\title{
Projet CHERLOC : Etude comparative d'un linéaire côtier protégé par des blocs artificiels à travers des approches sociétales, de biodiversité et d'ingénierie.
}

\author{
Dominique MOUAZE ${ }^{6}$, Etienne CUNGE ${ }^{1}$, Pascal CLAQUIN ${ }^{2}$, Agnès SALINAS $^{3}$, \\ Guillaume CARPENTIER ${ }^{4}$, Laurent QUENTRIC ${ }^{5}$, Cyril GIRAUDEL ${ }^{\mathbf{1}}$, \\ Osanne PAIREAU ${ }^{1}$, Sophie LEFEEZ $^{3}$, Maxime CORDELLIER ${ }^{3}$, Iman SAFARI $^{4}$, \\ Jean-Philippe PEZY ${ }^{6}$, Jean-Claude DAUVIN ${ }^{6}$
}

1. ARTELIA, 16 Rue Simone Veil, 93400 Saint-Ouen-Sur-Seine, France. etienne.cunge@arteliagroup.com; cyril.giraudel@arteliagroup.com; osanne.paireau@arteliagroup.com

2. Normandie Univ, UNICAEN, CNRS, BOREA, 14000 Caen, France. pascal.claquin@unicaen.fr

3. Normandie Univ, UNICAEN, CERREV, 14000 Caen, France. agnes.salinas@unicaen.fr; maxime.cordellier@unicaen.fr; sophie.lefeez@unicaen.fr

4. ESITC, 1 Rue Pierre et Marie Curie, 14610 Epron, France. guillaume.carpentier@esitc-caen.fr; iman.safari@esitc-caen.fr

5. MARC SA, 114 rue des Fougères, 50110 Tourlaville, France lquentric@marc-gw.fr

6. Normandie Univ, UNICAEN, UNIROUEN, M2C, 14000 Caen, France dominique.mouaze@unicaen.fr; jean-philippe.pezy@unicaen.fr; jean-claude.dauvin@unicaen.fr

\section{Résumé :}

L'objectif principal de ce projet est de créer et d'étudier deux sites pilotes en région Normandie en intégrant deux types de blocs artificiels à des digues existantes (Cherbourg, Ouistreham) à travers des approches d'acceptabilité sociale, d'amélioration de la biodiversité marine et d'ingénierie côtière. Des conditions hydrodynamiques sévères impliquent l'emploi de blocs de béton $\left(\mathrm{BCR}, \mathrm{ACCROPODE}^{\mathrm{TM}}, \ldots\right)$ à la place d'enrochements naturels afin d'assurer une stabilité hydraulique de l'ouvrage de protection et également de limiter les franchissements. Un bloc de carapace (DOUBLECUBE) a été développé au sein du laboratoire M2C possédant de nombreux atouts (performance, facilité de pose, multi usages) cependant il n'a pas encore atteint le stade de démonstrateur industriel. De même, ARTELIA a conçu une nouvelle génération de blocs de pied pour les digues à talus : les ACCROBERM ${ }^{\mathrm{TM}} \mathrm{II}$ qui se substitue à la butée de pied (stabilisation de la carapace) grâce à sa forme circulaire. Du fait de sa forme creuse, sa stabilité est également assurée par un remplissage à l'aide d'enrochements. Plusieurs avantages environnementaux émergent alors, i.e. emprise limitée sur les fonds 


\section{Thème 4 - Ouvrages portuaires, offshore et de plaisance}

marins, impacts environnementaux indirects limités, interactions avec la biodiversité. Sera réalisée une étude comparative de la biodiversité d'un linéaire côtier protégé par des enrochements artificiels ou naturels à travers des approches écosystémiques sur macroflore, macrofaune et biofilms de micro-algues. Le CERREV s'interroge sur les réactions des résidents locaux concernant une potentielle installation de ces blocs. Analyser l'acceptabilité de ces dispositifs par les populations locales bien en amont des phases critiques qui nécessitent des réactions d'urgence (risque d'inondation) permettrait d'envisager, pour les développeurs, un large panel d'adaptation de leurs dispositifs afin de construire avec les populations concernées les meilleures solutions.

\section{Mots-clés :}

Protection côtière, Blocs artificiels, Biodiversité, Approche écosystémique, Acceptabilité sociale, Remédiation.

\section{Introduction}

Les paysages côtiers mondiaux sont en constante transformation par la construction d'infrastructures (digues, jetées) afin de répondre à des demandes croissantes d'activités économiques, résidentielles, ... L'environnement naturel côtier est morcelé par les constructions humaines empiétant sur les habitats écologiques des zones intertidales. Ce caractère est particulièrement marqué sur les côtes de la Manche pour lesquelles le marnage est élevé. Encore récemment, la réponse à un événement de submersion marine était abordée sous un angle d'ingénierie pure avec en réponse la construction d'une nouvelle infrastructure. Depuis une dizaine d'années, un changement de paradigme voit le jour en intégrant la nature et la société dans l'approche technique des projets côtiers. Au Pays-Bas, le concept 'Building with Nature' émerge à travers une expérience grandeur nature de très grande ampleur (Sand Motor) afin de protéger un linéaire côtier à l'aide d'un rechargement sableux à grand volume et d'apporter une réponse 'douce' et long terme au problème d'érosion du littoral. En Europe du Nord, par exemple, des stratégies de 'zone tampon' permettent la construction progressive de retenues sédimentaires et de zones humides, favorables à l'écosystème, et jouant le rôle de protection jusqu'à la prochaine tempête centennale. Les variables et les conditions du système environnemental sont intégrées dès les premières réflexions du projet en tenant compte des changements long terme à la limite de l'échelle de temps de l'ingénierie. Ici, les 'services' de l'écosystème sont déjà de grandes ampleurs par la fonction d'atténuation de la submersion marine mais aussi par la création de zones de loisirs et de récréation, par la formation d'un écosystème riche sur la frange littoral, par la préservation des nappes phréatiques d'eau douce.

Concernant les infrastructures côtières, les facteurs de risque de diminution de la biodiversité locale ont été mis en avant par BULLERI et CHAPMAN (2004; 2010). De même sont pointés, la relative toxicité de ces structures en béton, la rupture de continuité du linéaire côtier ainsi que les impacts indirects comme le prélèvement de sable de 


\section{XVI'̀mes Journées Nationales Génie Côtier - Génie Civil \\ Le Havre 2020}

construction, d'enrochements de carrière ou de production de dioxyde de carbone (MOSCHELLA et al., 2005; TERLIZZI \& FAIMALI, 2010). Plusieurs études (GLASBY et al., 2007 ; FIRTH et al., 2014) mettent en garde contre le développement possible d'espèces introduites à caractère invasif et la modification des schémas naturels de dispersion des espèces. L'ensemble de ces études citées précédemment a induit un changement de paradigme de la part de l'ingénierie côtière considérant maintenant les aspects environnementaux au même rang que les besoins techniques. Le nombre de plus en plus important d'études sur ces sujets montre que les impacts restent relativement limités malgré la forte anthropisation des côtes. Dans le cadre du projet CHERLOC (2020-2022), une comparaison de la biodiversité (biofilms de micro algues, microflore, macrofaune) d'une côte protégée par des enrochements artificiels ou naturels sera réalisée à travers d'approches écosystémiques. Trois types de substrats seront examinés : substrats durs naturels, structures artificielles en place et nouvelles structures installées pendant le projet. Récemment, EVANS et al. (2016) montrent que l'amélioration de la biodiversité était dépendante de la composition des bétons, de la rugosité de surface et de la présence d'anfractuosités. De même l'altération (action des vagues, climat, érosion chimique) de ces structures en béton peut être freinées par le biofouling (PERKOL-FINKEL \& SELLA, 2015). Ce projet s'inscrit ainsi dans une démarche cherchant à remplir les besoins de dimensionnement de l'ingénierie (franchissements, stabilité, contrôle sédimentaire) tout en préservant voire améliorant l'habitat écologique de ces zones connues pour leur relative pauvreté en terme d'espèces pouvant vivre dans les hauts niveaux des estrans. Dans sa localisation, le tapis d'enrochements artificiels n'aura pas d'impacts sur la qualité des eaux de baignade. Les périodes de pose et de dépose seront réalisées en dehors des périodes de baignades estivales.

Comme précisé dans le paragraphe précédent, l'implantation des blocs dans cette zone vise à :

- quantifier les effets sur le transport sédimentaire,

- caractériser les blocs en tant que support (quantité, qualité) de biodiversité marine.

Les résultats enrichiront également la connaissance de la diversité marine normande et la prise de décision d'aménagements pour améliorer la biodiversité et la production des environnements côtiers. L'étude d'acceptabilité sociale du laboratoire CERREV a pour objectif d'évaluer les facteurs influençant l'appréciation collective des blocs de béton pour protéger les littoraux, en vue de faciliter l'insertion de ces blocs dans le paysage côtier. Elle vise à permettre une intégration harmonieuse du projet dans les communautés côtières ciblées en favorisant sa co-construction avec les parties prenantes. Cette démarche doit être vue pour les porteurs de l'étude comme une occasion d'optimiser leur projet d'anticipation des risques, et pour les riverains comme une occasion d'être écoutés et impliqués dans le choix et la mise en œuvre d'une méthode de protection de leur littoral. Le questionnement portera sur plusieurs aspects : esthétique, environnemental, sentiment de sécurité, représentations (dimension patrimoniale à Cherbourg), interactions avec les 


\section{Thème 4 - Ouvrages portuaires, offshore et de plaisance}

usages courants. L'étude conduite par le CERREV portera sur plusieurs types de public (habitants d'une zone littorale, élus locaux, institutions de promotion du tourisme local, compagnies de Ferries, pêcheurs côtiers, professionnels et amateurs, plaisanciers, promeneurs, ...). L'objectif est de mieux cerner les représentations sociales et les réactions comportementales et psychosociales devant la proposition de blocs de protection des sites littoraux de la Région, de pouvoir expliquer et discuter des choix techniques de protection face aux risques de submersion, et d'aider les populations à comprendre et se représenter l'action de ces blocs sur l'environnement marin et leur lieu de vie. Plus largement, l'étude fournira un instantané de la perception du changement climatique et des opinions quant à ses conséquences sur les territoires habités. Ce projet multidisciplinaire implique une entreprise régionale de travaux maritimes (MARC SA), un bureau d'étude en ingénierie (ARTELIA), une école d'ingénieurs (ESITC Caen), trois laboratoires de recherche de l'Université de Caen Normandie (CERREV, BOREA, M2C), Ports de Normandie et le CEREMA.

\section{Les deux sites étudiés en Manche : Digue de l'Est (Cherbourg), Epi du chenal de Ouistreham}

\subsection{Cas de la digue de 1'Est de Cherbourg}

La grande rade artificielle de Cherbourg (1500ha) est protégée à l'est par la digue de l'Est. Cette dernière est caractérisée comme mixte (ouvrage maçonné vertical monté sur un soubassement en talus) et arasée à la cote $+6.5 \mathrm{CM}$, ce qui la rend submersible à marée haute. L'implantation du projet, sur deux linéaires de $30 \mathrm{~m}$ de part et d'autre de l'ouvrage vertical, est située à $420 \mathrm{~m}$ du fort de l'île Pelée (photo 1). Le niveau de surcote associé à une houle centennale est de $+7.5 \mathrm{mCM}$. Les directions des vents dominants sont de secteur Sud-Ouest et Nord-Est. Les houles centennales de Nord-Est sont normales à la digue de l'Est et peuvent atteindre un $\mathrm{H}_{\mathrm{s}}$ de $5 \mathrm{~m}$ pour une période de $10 \mathrm{~s}$. Les houles centennales d'Ouest peuvent atteindre les mêmes niveaux de houle significative cependant l'obliquité sera élevée $\left(\sim 30^{\circ}\right)$ ainsi que la période pic $(\sim 12 \mathrm{~s})$. Le pré-dimensionnement des DOUBLE-CUBE $(5 \mathrm{t})$ et des ACCROBERM $^{\mathrm{TM}} \mathrm{II}(2 \mathrm{t})$ a été réalisé à l'aide de la formulation de Hudson dans un premier temps et en prenant en compte l'obliquité. La pente de la carapace a été fixée à 4/3 (figure 1). Les dommages (déplacements des blocs) sur la carapace seront évalués à l'aide du croisement de différentes techniques d'observations (LIDAR, SfM, InSAR). 


\section{XVİ̀mes Journées Nationales Génie Côtier - Génie Civil \\ Le Havre 2020}

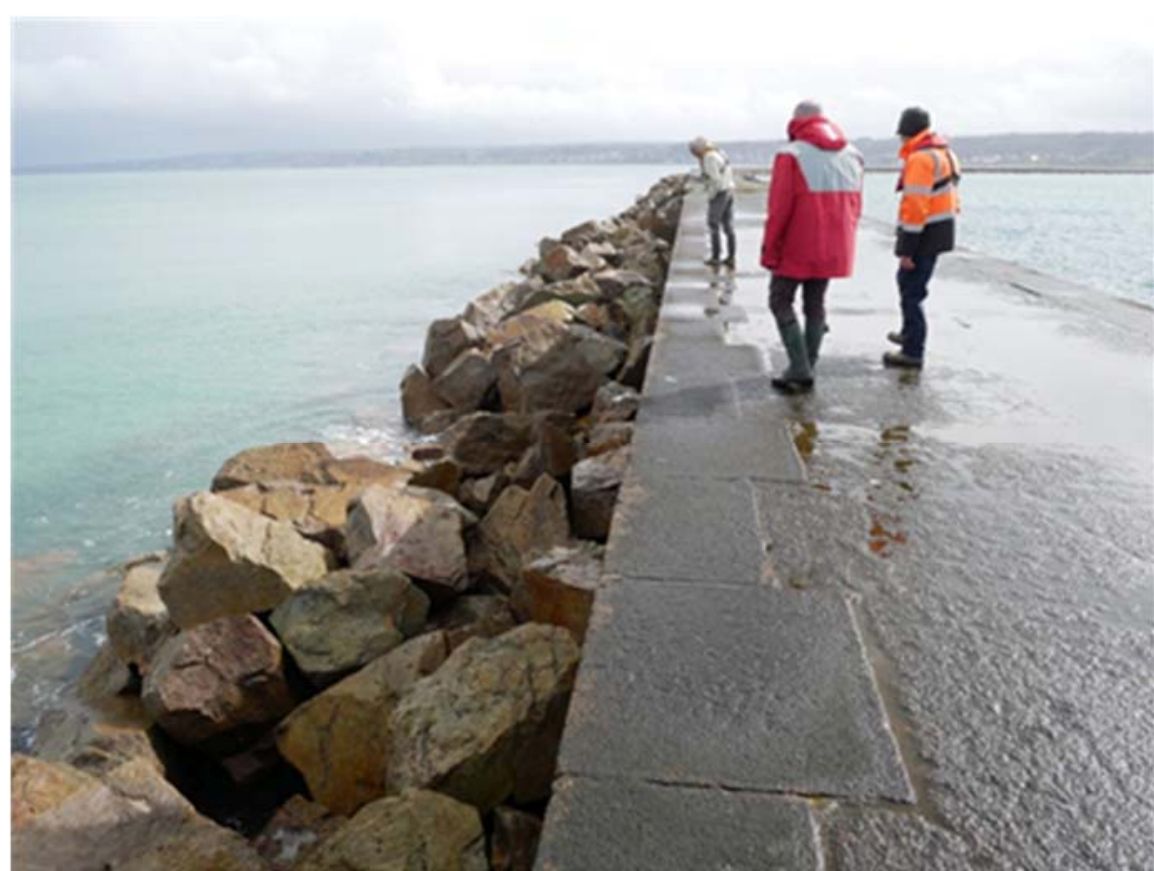

Photo 1. Vue des enrochements naturels existants de la digue de l'Est.

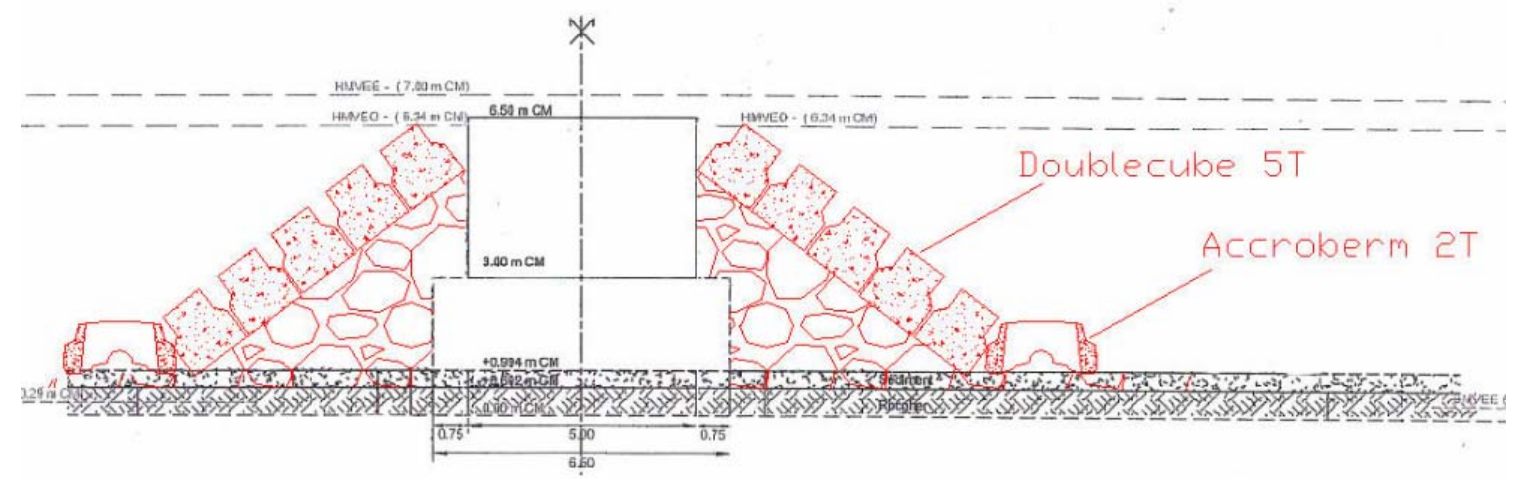

Figure 1. Coupe des carapaces et butées de pied de part et d'autre de la digue de l'Est.

\subsection{Cas de l'épi du chenal d'accès au port de Ouistreham}

Le deuxième site d'implantation est situé à l'ouest de l'épi qui borde le chenal d'accès du port de Ouistreham et à l'extrémité de la plage Riva-Bella. La zone de travaux est implantée sur le domaine public maritime de Ports de Normandie. Les vents de secteur Ouest à Sud-Ouest sont dominants. Les houles centennales peuvent atteindre une hauteur significative de $5.8 \mathrm{~m}$ pour une période pic de $9 \mathrm{~s}$. La réfraction bathymétrique ainsi que la diffraction autour de l'épi impliquent une direction normale des vagues vis à vis du tapis d'enrochements artificiels (photo 2). La dérive littorale induit un transport sédimentaire d'Ouest en Est qui provoque une zone d'accrétion en amont (Ouest) de l'épi (encart photo 2). 


\section{Thème 4 - Ouvrages portuaires, offshore et de plaisance}

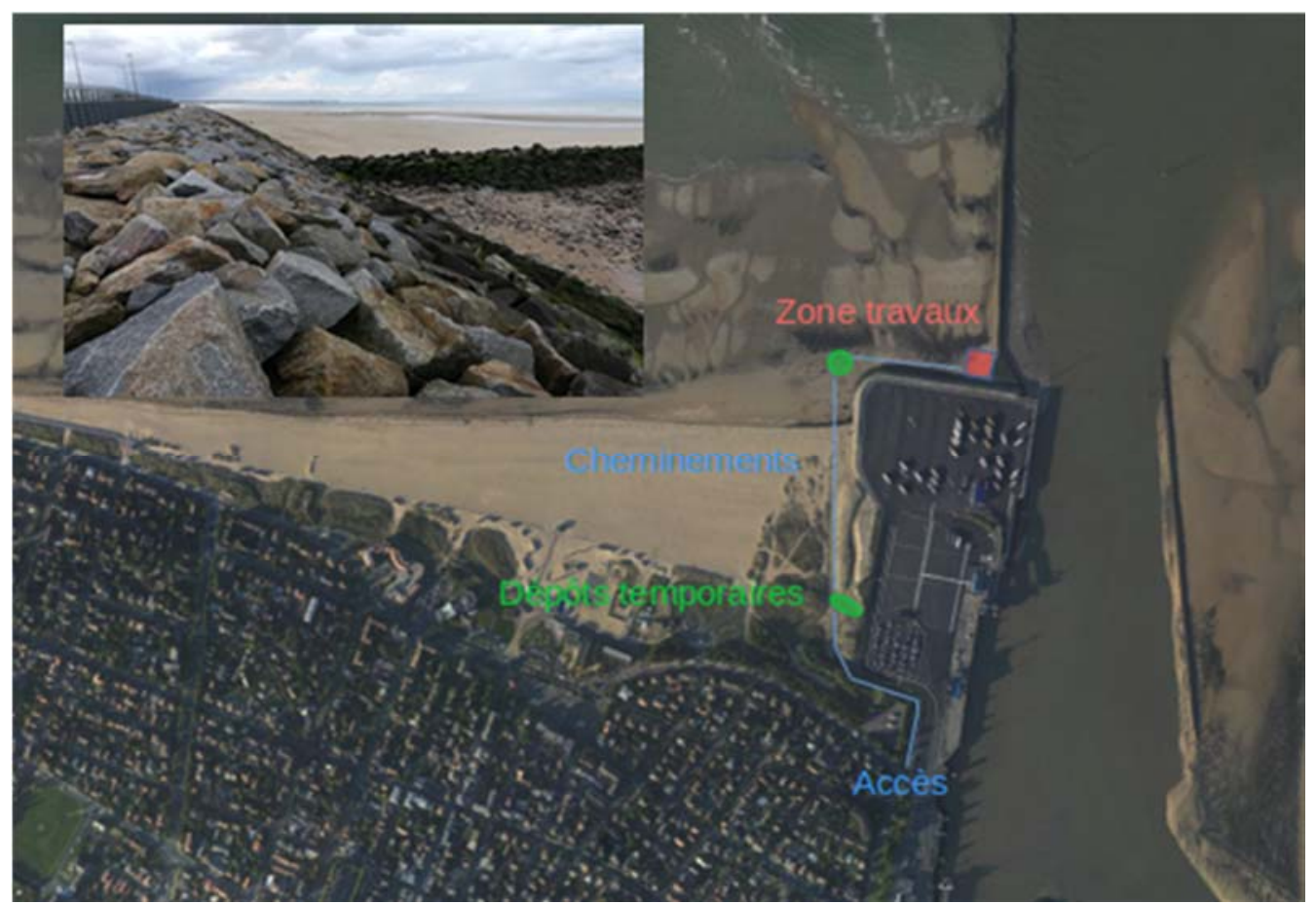

Photo 2. Vue aérienne des zones d'accès, de dépôts et de travaux près de l'épi (encart).

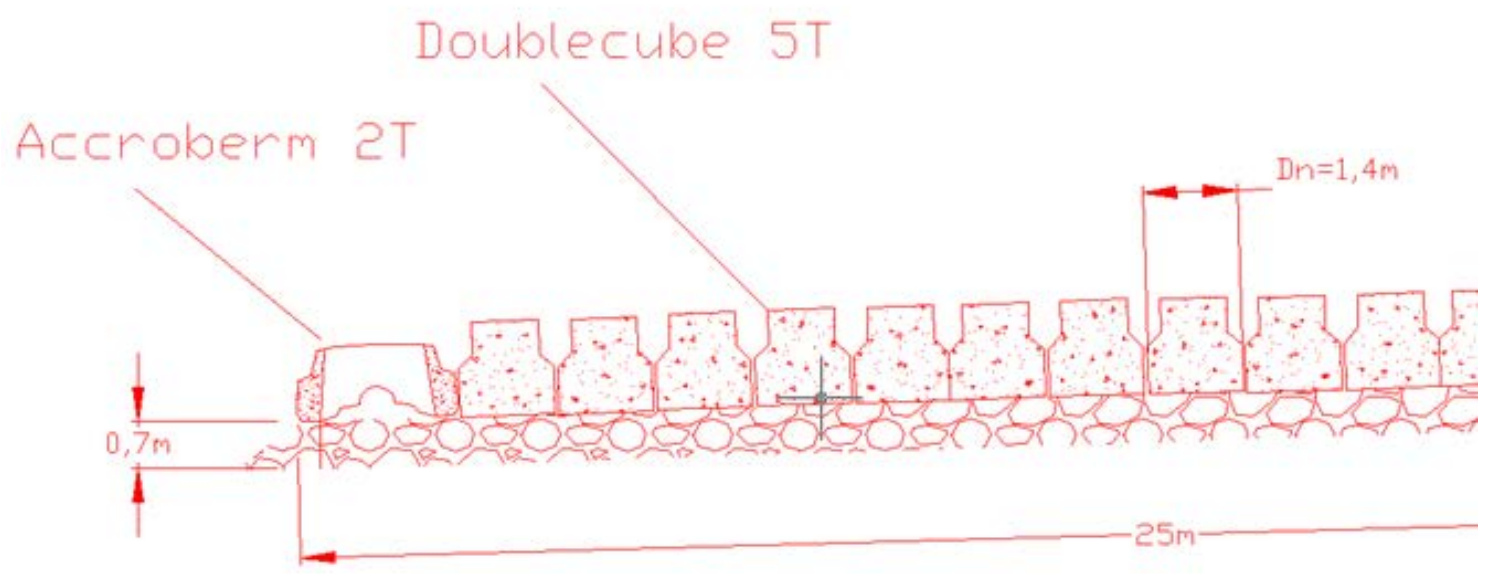

Figure 2. Coupe du tapis d'enrochements artificiels positionnés en haut estran près de l'épi du chenal d'accès au port de Ouistreham.

La présence du tapis d'enrochements artificiels (figure 2) va modifier très localement (emprise de $25 \mathrm{~m} \times 25 \mathrm{~m}$ ) la propagation des vagues et des courants :

- diffraction par contournement de la zone,

- réflexion locale en périphérie de la zone,

- génération du déferlement par rupture bathymétrique,

- dissipation d'énergie par frottement sur les éléments rugueux et par aération.

Ces modifications sont voulues et ont pour rôle de modifier les dépôts sédimentaires afin de retarder l'accrétion en périodes de faible énergie des vagues et des courants et d'éroder 


\section{XVİ̀mes Journées Nationales Génie Côtier - Génie Civil \\ Le Havre 2020}

les dépôts en périodes de forte énergie afin de limiter les zones de concentration par "étalement" sur l'ensemble de l'estran. Les conséquences à moyen terme sont de retarder les franchissements sédimentaires de l'épi longeant le chenal d'accès et de favoriser une répartition homogène des sédiments. Cette répartition homogène doit modifier le profil de plage afin d'initier plus rapidement un déferlement glissant et de dissiper l'énergie des vagues de manière progressive. La complexité des processus hydro-sédimentaires empêchent de prédire avec certitudes les phénomènes décrits plus haut cependant cette étude à échelle 1 permettra d'apporter des connaissances réelles afin d'améliorer les modèles numériques par exemple. Cette étude reste expérimentale (démonstrateur), d'une durée limitée (deux ans) et réversible.

La présence du tapis d'enrochement va modifier principalement la fraction grossière des sédiments en transit le long du littoral. La partie fine va continuer à dériver en suspension. La zone du projet étant située en amont des éventuelles sources de pollution (station d'épuration, dragage, zones de dépôts,...), elle n'aura pas d'incidences sur la modification de la qualité des eaux littorales.

\section{Les blocs artificiels, approche d'ingénierie côtière}

\subsection{Le bloc de butée de pied ACCROBERM ${ }^{\mathrm{TM}} \mathrm{II}$}

L'ACCROBEM ${ }^{\mathrm{TM}}$ II (figure 3), conçu par ARTELIA, est un bloc de forme cylindrique et rempli d'enrochements naturels dont les fonctionnalités sont multiples :

- stabilité de la butée de pied,

- support de biodiversité en offrant de nouveaux habitats, y compris des micro-habitats.

La grande stabilité de ce bloc est assurée par sa forme annulaire, son centre de gravité bas et ses évents. Ce bloc est rempli d'enrochements ou de matériaux permettant d'être attractif pour les larves, les juvéniles mais aussi les adultes créant un nouvel écosystème ou recréant l'écosystème préexistant.

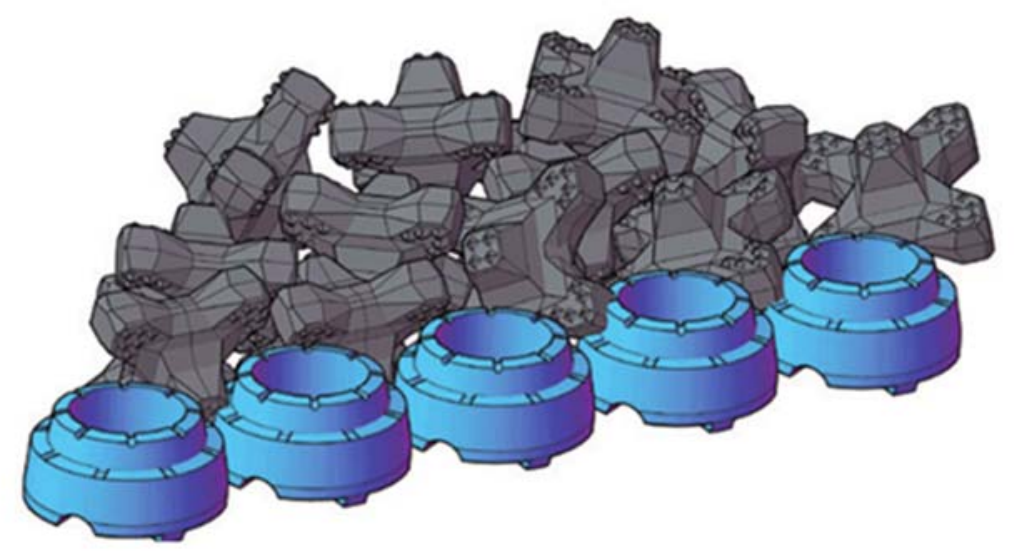

Figure 3. Vue 3D d'une carapace d'ACROPODE s'appuyant sur une butée de pied en ACCROBERM ${ }^{T M} I I$. 


\section{Thème 4 - Ouvrages portuaires, offshore et de plaisance}

Ce bloc améliore également la constructibilité et la mise en place avec des moyens techniques maritimes classiques. Son ancrage au sol, la position de son centre de gravité ainsi que sa porosité élevée permettent une stabilisation pérenne dans les zones de déferlement. La suppression de la plupart des souilles en rocher ou de la butée en enrochements offre une solution économique et facile à mettre en œuvre tout en réduisant l'emprise des ouvrages sur le domaine maritime. De plus cette solution technique peut s'intégrer à un site écologiquement sensible en offrant des possibilités d'écoconception de haute valeur ajoutée. La biodiversité des blocs qui seront installés consistera à inventorier l'ensemble des espèces de la flore et de la faune qui coloniseront les blocs. Les communautés présentes sur les substrats durs naturels localisées à proximité seront comparées avec les différentes successions écologiques observées sur les blocs durant l'expérience de 2 ans. Une attention particulière sera portée sur la faune vagile qui sera présente parmi les blocs et sur la présence d'espèces non-indigènes.

\subsection{Le bloc de carapace DOUBLE-CUBE}

Les enseignements issus des travaux sur le STARBLOC (SAFARI, 2011 ; SAFARI et al., 2012 ; SAFARI et al., 2018) ont permis de concevoir (ROPERT, 2017) et de tester en canal et bassin un nouveau type de bloc artificiel, le DOUBLE-CUBE. En effet, les travaux novateurs sur la variabilité de la perméabilité au sein de la carapace ont montré qu'un plan de pose fortement imbriqué (type bloc massif STARBLOC) conduit à une chute de perméabilité induisant une rupture des écoulements à travers la carapace. Ce phénomène hydraulique implique une augmentation du franchissement lors du run-up et surtout des sous-pressions lors de la vidange de la nappe de la digue poreuse lors du rundown. Ces sous-pressions conduisent à des soulèvements et extractions en masse des blocs de carapaces. Il est donc indispensable d'assurer un minimum de perméabilité quel que soit le plan de pose utilisé pour un bloc artificiel. C'est le cas du DOUBLE-CUBE qui offre un nombre de plan de pose limité tout en garantissant la stabilité.
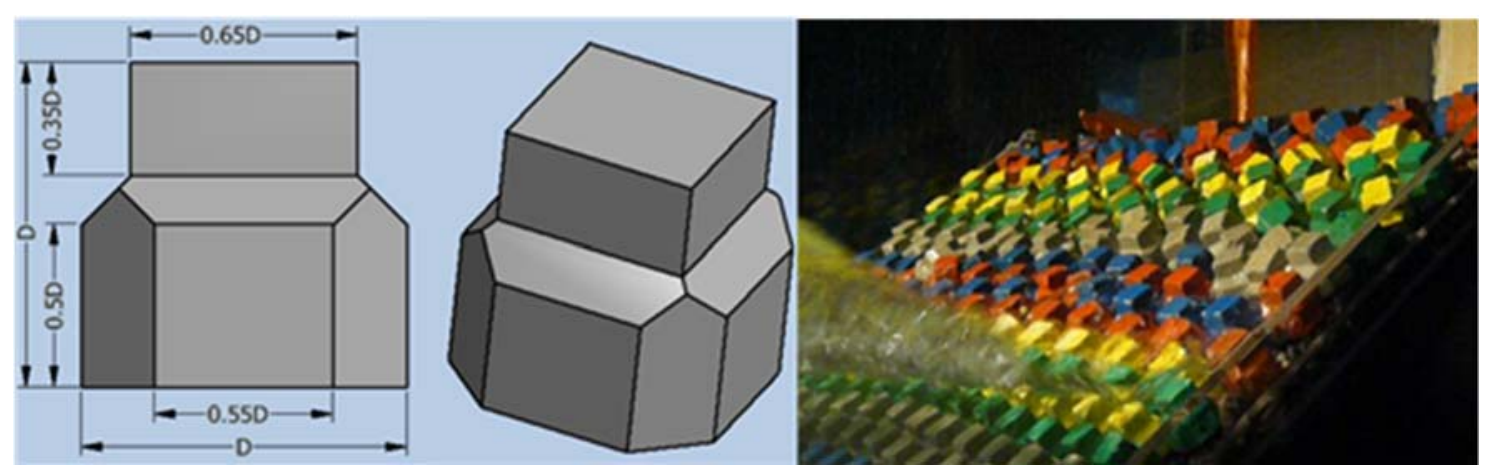

Figure 4. Géométries du DOUBLE-CUBE et vue des tests en canal à houle.

Ce bloc présente une base cubique chanfreinée (figure 4) ainsi qu'une partie supérieure de section cubique d'arête $65 \%$ plus petite que la base. Cette rugosité superficielle induite 


\section{XVI èmes Journées Nationales Génie Côtier - Génie Civil \\ Le Havre 2020}

va limiter les franchissements par dissipation turbulente et aération (cisaillement important des vitesses). Le placement 'face contre face' optimise le frottement surfacique et conduit à une stabilité optimale malgré une imbrication absente (recouvrement nul). Le nombre de stabilité $\mathrm{N}_{\mathrm{S}}$ atteint 2.9 alors que le coefficient de stabilité $\mathrm{K}_{\mathrm{D}}$, selon Hudson, monte jusqu'à 18 (en canal à houle).

La forme pseudo-hexagonale de la base conduit également à un phénomène de clé de voûte permettant de conserver la stabilité des blocs adjacents à un bloc extrait de la carapace. Il n'y a pas d'effet de 'détricotage' comme observé sur des blocs artificiels imbriqués.

\section{Conclusion}

L'objectif principal de ce projet (2020-2022) est de créer et d'étudier deux sites pilotes en région Normandie en intégrant deux types de blocs artificiels à des digues existantes (Cherbourg, Ouistreham) à travers des approches d'acceptabilité sociale, de biodiversité et d'ingénierie côtière. Les autorisations (AOT, DDTM14, DDTM50) ont été accordées en juin 2020 et les phases travaux maritimes sont prévues en décembre 2020 (Ouistreham) puis au printemps 2021 (Cherbourg). Les essais en modèle physique sont prévus pendant l'été 2020 et les premières observations écologiques en octobre 2020 puis un suivi des deux sites avec une stratégie à 3/6/12 mois. Les questionnaires de l'étude sociétale sont en cours de rédaction.

\section{Références}

BULLERI F., CHAPMAN M.G. (2004). Intertidal assemblages on artificial and natural habitats in marinas on the north-west coast of Italy. Marine Biology, Vol. 145(2), pp 381-391. https://doi.org/10.1007/s00227-004-1316-8

BULLERI F., CHAPMAN M.G. (2010). The introduction of coastal infrastructure as a driver of change in marine environments. Journal of Applied Ecology, Vol. 47(1), pp 26 35. https://doi.org/10.1111/j.1365-2664.2009.01751.x

EVANS A.J., FIRTH L.B., HAWKINS S.J., MORRIS E.S., GOUDGE H., MOORE P.J. (2016). Drill-cored rock pools: an effective method of ecological enhancement on artificial structures. Marine and Freshwater Research, Vol. 67(1), pp 123-130. https://doi.org/10.1071/MF14244

FIRTH L.B., THOMPSON R.C., BOHN K., ABBIATI M., AIROLDI L., BOUMA T. J., FERRARIO F. (2014). Between a rock and a hard place: environmental and engineering considerations when designing coastal defence structures. Coastal Engineering, Vol. 87, pp 122-135. https://doi.org/10.1016/j.coastaleng.2013.10.015

GLASBY T.M., CONNELL S.D., HOLLOWAY M.G., HEWITT C.L. (2007). Nonindigenous biota on artificial structures: could habitat creation facilitate biological invasions? Marine biology, Vol. 151(3), pp 887-895. https://doi.org/10.1007/s00227-006-0552-5 


\section{Thème 4 - Ouvrages portuaires, offshore et de plaisance}

MOSCHELlA P.S., ABBIATI M., ÅBERG P., AIROLDI L., ANDERSON J.M., BACCHIOCCHI F., GRANHAG L. (2005). Low-crested coastal defence structures as artificial habitats for marine life: using ecological criteria in design. Coastal Engineering, Vol. 52(10-11), pp 1053-1071. https://doi.org/10.1016/j.coastaleng.2005.09.014

PERKOL-FINKEL S., SELLA I. (2015). Harnessing urban coastal infrastructure for ecological enhancement. In Proceedings of the Institution of Civil Engineers-Maritime Engineering, Vol. 168(3), pp 102-110. https://doi.org/10.1680/jmaen.15.00017

ROPERT F. (2017). Structure for protecting sea and/or river construction work, and protective block used. Brevet TN2OI 5000422 (AI) 2017-01-03.

SAFARI I. (2011). Analyse de la performance hydraulique d'un nouveau type de bloc artificiel utilise pour la protection côtière. Thèse de l'Université de Caen, 270 p. https://tel.archives-ouvertes.fr/tel-00705242

SAFARI I., MOUAZÉ D., ROPERT F., HAQUIN S., EZERSKY A. (2012). Influence du plan de pose sur les distributions de porosité au sein d'une carapace de digue à talus. XIIème Journées Nationales Génie Côtier Génie Civil Cherbourg, pp 791-798. https://doi.org/10.5150/jngcgc.2012.086-S

SAFARI I., MOUAZÉ D., ROPERT F., HAQUIN S., EZERSKY A. (2018). Hydraulic stability and wave overtopping of Starbloc $\AA$ armored mound breakwaters. Ocean Engineering, Vol. 151, pp 268-275. https://doi.org/10.1016/j.oceaneng.2017.12.061

TERLIZZI A., FAIMALI M. (2010). Fouling on artificial substrata. Biofouling, pp 170-184. https://doi.org/10.1002/9781444315462.ch12 